\title{
A CHARACTERIZATION OF THE FOUR CHEBYSHEV ORTHOGONAL FAMILIES
}

\author{
E. BERRIOCHOA, A. CACHAFEIRO, AND J. M. GARCIA-AMOR \\ Received 26 September 2004 and in revised form 2 June 2005
}

We obtain a property which characterizes the Chebyshev orthogonal polynomials of first, second, third, and fourth kind. Indeed, we prove that the four Chebyshev sequences are the unique classical orthogonal polynomial families such that their linear combinations, with fixed length and constant coefficients, can be orthogonal polynomial sequences.

\section{Introduction}

The classical orthogonal polynomials (OP) on the real line, which are the most useful and important families, can be characterized by different conditions (see [3]). In the case of bounded support, the classical model is the family of Jacobi polynomials, which has been extensively studied. Very well-known families of Jacobi polynomials are the so-called Chebyshev polynomials of first, second, third, and fourth kind (see [9]).

The orthogonal polynomials with respect to rational modifications of these Chebyshev weights are the Bernstein polynomials (see $[3,9]$ ). It is well known that the Bernstein polynomials can be expressed as linear combinations of Chebyshev polynomials, with fixed length and constant coefficients (see $[4,5]$ ). This property of orthogonality satisfied by the linear finite combinations of sequences of orthogonal polynomials is quite general. Indeed, taking into account the result of Uvarov (see [10]), the orthogonal polynomial sequences with respect to a rational modification of a positive measure on an interval can be written as a linear combination of the orthogonal polynomials with respect to the original measure, with fixed length and coefficients depending on the degrees. Although the representation of the Bernstein polynomials is a consequence of Uvarov's result, it is important to note that in this first case, the coefficients are constant.

The study of linear combinations of orthogonal polynomials is an interesting subject for different reasons. For example, several quadrature formulas have been obtained choosing the nodal points as the zeros of some linear combinations. Another reason could be the important role that these linear combinations of OP play in the problem of linearization of products of $\mathrm{OP}$, as well as, in the problem of approximation of the polynomial solution for differential equations. For a detailed presentation of these problems and some others, see [6]. 
The general problem of the orthogonality for combinations of length three was studied in [2]. Indeed, there necessary and sufficient conditions on the sequences of coefficients of the linear combinations with fixed length three were obtained in order that the new sequences be orthogonal. Very interesting examples appear in [2] and also in [1]. Another interesting contribution in the same direction is [7], in which the authors study the orthogonality of linear combinations of two orthogonal polynomial sequences. Applying the results in $[2,7]$, we have studied in [4] the following inverse problem, that is, we have determined that the four Chebyshev sequences are the unique Jacobi sequences such that their linear combinations with length 2 or 3 and constant coefficients can be orthogonal.

Our aim in this paper is to prove that the four Chebyshev families are the unique families among all the classical orthogonal polynomials such that linear finite combinations with arbitrary fixed length and constant coefficients can be orthogonal sequences. However, this property can be satisfied by nonclassical orthogonal polynomial sequences. Indeed, we give examples of nonclassical orthogonal polynomial sequences such that linear combinations, with fixed length and constant coefficients, are orthogonal.

The structure of the paper is the following. In Section 2, first we recall some properties concerning the classical orthogonal polynomials and we present the Bernstein orthogonal polynomials. Moreover, we construct several examples of finite linear combinations, with constant coefficients, of nonclassical orthogonal polynomials, such that they are again orthogonal sequences. Finally, in Section 3, we prove our main result, that is, the property that characterizes the four Chebyshev orthogonal polynomial sequences.

\section{The Bernstein polynomials}

In the positive definite case, the classical orthogonal polynomials are the Jacobi polynomials $P_{n}^{(\alpha, \beta)}(x)$ which are orthogonal with respect to the positive measure on $[-1,1]$, $d \mu_{\alpha, \beta}(x)=(1-x)^{\alpha}(1+x)^{\beta} d x$, with $\alpha, \beta>-1$, the Sonine-Laguerre polynomials $L_{n}^{(\alpha)}(x)$ orthogonal with respect to the positive measure on $[0,+\infty), d \mu_{\alpha}(x)=x^{\alpha} e^{-x} d x$, with $\alpha>$ -1 , and the Hermite polynomials $H_{n}(x)$ orthogonal with respect to the positive measure on $(-\infty,+\infty), d \mu(x)=e^{-x^{2}} d x$.

By virtue of their orthogonality, the classical monic polynomials satisfy the following three-term recurrence relations:

$$
P_{n}^{(\alpha, \beta)}(x)=\left(x-\beta_{n-1}\right) P_{n-1}^{(\alpha, \beta)}(x)-\gamma_{n-1} P_{n-2}^{(\alpha, \beta)}(x), \quad n \geq 1,
$$

where

$$
\begin{gathered}
\beta_{n}=\frac{\beta^{2}-\alpha^{2}}{(2 n+\alpha+\beta)(2 n+\alpha+\beta+2)}, \\
\gamma_{n}=\frac{4 n(n+\alpha)(n+\beta)(n+\alpha+\beta)}{(2 n+\alpha+\beta)^{2}(2 n+\alpha+\beta+1)(2 n+\alpha+\beta-1)},
\end{gathered}
$$

with $P_{0}(x)=1$ and $P_{-1}(x)=0\left(\beta_{0}=-\alpha\right.$ in case $\left.\alpha+\beta=0\right)$;

$$
L_{n}^{(\alpha)}(x)=\left(x-\beta_{n-1}\right) L_{n-1}^{(\alpha)}(x)-\gamma_{n-1} L_{n-2}^{(\alpha)}(x), \quad n \geq 1,
$$


where

$$
\beta_{n}=2 n+\alpha-1, \quad \gamma_{n}=(n-1)(n+\alpha-1),
$$

with $L_{0}^{(\alpha)}(x)=1$ and $L_{-1}^{(\alpha)}(x)=0$;

$$
H_{n}(x)=x H_{n-1}(x)-\gamma_{n-1} H_{n-2}(x), \quad n \geq 1,
$$

where

$$
\gamma_{n}=\frac{1}{2}(n-1)
$$

with $H_{0}(x)=1$ and $H_{-1}(x)=0$.

Notice that the unique classical families with constant coefficients in the three-term recurrence relation are those Jacobi families corresponding to $\alpha= \pm 1 / 2$ and $\beta= \pm 1 / 2$, that is, the Chebyshev families. Indeed, they satisfy for $n \geq 2$ the same recurrence relation $P_{n}(x)=x P_{n-1}(x)-(1 / 4) P_{n-2}(x), n \geq 2$, with different initial conditions.

The Bernstein polynomials generalize the classical Chebyshev polynomials. They are orthogonal with respect to positive measures obtained by introducing a positive polynomial divisor into the Chebyshev weight function (see $[3,5,9])$. Next, we recall a useful representation for these Bernstein sequences.

Let $q_{k}(x)$ be a polynomial with real coefficients and positive in $[-1,1]$. By Féjer-Riesz representation, there exists a polynomial $A_{k}(z)=\sum_{i=0}^{k} m_{i} z^{i}$ such that $A_{k}(z) \neq 0$ for $|z| \leq$ $1, A_{k}(0)>0$ and $q_{k}(\cos \theta)=\left|A_{k}\left(e^{i \theta}\right)\right|^{2}$.

If $d \mu_{1}(x)$ is the rational modification of the Chebyshev measure of first kind $d \mu_{1}(x)=$ $d x /\left(\pi q_{k}(x) \sqrt{1-x^{2}}\right)$, and $\left\{P_{n}\left(x, \mu_{1}\right)\right\}$ is the monic orthogonal polynomial sequence with respect to $\mu_{1}\left(\operatorname{MOPS}\left(\mu_{1}\right)\right)$, then for $n \geq k$,

$$
P_{n}\left(x, \mu_{1}\right)=\dot{T}_{n}(x)+\sum_{i=1}^{k} \frac{m_{i}}{m_{0}} \frac{1}{2^{i}} \dot{T}_{n-i}(x),
$$

where $\left\{\dot{T}_{n}(x)\right\}$ is the sequence of monic Chebyshev polynomials of first kind.

If $d \mu_{2}(x)$ is the rational modification of the Chebyshev measure of second kind $d \mu_{2}(x)=2 \sqrt{1-x^{2}} d x /\left(\pi q_{k}(x)\right)$, and $\left\{P_{n}\left(x, \mu_{2}\right)\right\}$ is the $\left(\operatorname{MOPS}\left(\mu_{2}\right)\right)$, then for $n \geq k$,

$$
P_{n}\left(x, \mu_{2}\right)=\dot{U}_{n}(x)+\sum_{i=1}^{k} \frac{m_{i}}{m_{0}} \frac{1}{2^{i}} \dot{U}_{n-i}(x),
$$

where $\left\{\dot{U}_{n}(x)\right\}$ is the sequence of monic Chebyshev polynomials of second kind.

If $d \mu_{3}(x)$ is the rational modification of the Chebyshev measure of third kind $d \mu_{3}(x)=$ $1 /\left(\pi q_{k}(x)\right) \sqrt{(1+x) /(1-x)} d x$, and $\left\{P_{n}\left(x, \mu_{3}\right)\right\}$ is the (MOPS $\left.\left(\mu_{3}\right)\right)$, then for $n \geq k$,

$$
P_{n}\left(x, \mu_{3}\right)=\dot{W}_{n}(x)+\sum_{i=1}^{k} \frac{m_{i}}{m_{0}} \frac{1}{2^{i}} \dot{W}_{n-i}(x),
$$

where $\left\{\dot{W}_{n}(x)\right\}$ is the sequence of monic Chebyshev polynomials of third kind. 
If $d \mu_{4}(x)$ is the rational modification of the Chebyshev measure of fourth kind $d \mu_{4}(x)=1 /\left(\pi q_{k}(x)\right) \sqrt{(1-x) /(1+x)} d x$, and $\left\{P_{n}\left(x, \mu_{4}\right)\right\}$ is the $\left(\operatorname{MOPS}\left(\mu_{4}\right)\right)$, then for $n \geq k$,

$$
P_{n}\left(x, \mu_{4}\right)=\dot{V}_{n}(x)+\sum_{i=1}^{k} \frac{m_{i}}{m_{0}} \frac{1}{2^{i}} \dot{V}_{n-i}(x),
$$

where $\left\{\dot{V}_{n}(x)\right\}$ is the sequence of monic Chebyshev polynomials of fourth kind.

Hence, it is clear that these types of linear combinations of Chebyshev polynomials are again orthogonal sequences. Although our aim is to prove that this property characterizes these Chebyshev families among all classical families, nevertheless, this property can be satisfied by nonclassical families. Next we present examples of nonclassical sequences such that they satisfy the same above property as the Chebyshev families.

Example 2.1. Let $\left\{P_{n}(x)\right\}$ be the MOPS satisfying the recurrence relation

$$
P_{n}(x)=\left(x-\epsilon_{n-1}\right) P_{n-1}(x)-\eta_{n-1} P_{n-2}(x), \quad n \geq 1,
$$

with $P_{-1}(x)=0$ and $P_{0}(x)=1$, and such that $\eta_{n}=a \epsilon_{n}$, with $a \in \mathbb{R}, a \neq 0$, and $\epsilon_{n} \neq 0$, for all $n$. Then the sequence of monic polynomials $\left\{Q_{n}(x)\right\}$ defined by

$$
Q_{n}(x)=P_{n}(x)+a P_{n-1}(x)
$$

is the MOPS with respect to a regular linear functional.

It is clear that the sequence $\left\{P_{n}(x)\right\}$ is different from the Chebyshev families. Besides, it is easy to see that the sequence $\left\{Q_{n}(x)\right\}$ satisfies the following three-term recurrence relation:

$$
Q_{n}(x)=\left(x-b_{n-1}\right) Q_{n-1}(x)-c_{n-1} Q_{n-2}(x), \quad \text { for } n \geq 0,
$$

with $c_{n}=a \epsilon_{n-1}$ and $b_{n}=\epsilon_{n}$. Indeed,

$$
\begin{aligned}
Q_{n}(x) & =P_{n}(x)+a P_{n-1}(x) \\
& =\left(x-\epsilon_{n-1}\right) P_{n-1}(x)-\eta_{n-1} P_{n-2}(x)+a\left(\left(x-\epsilon_{n-2}\right) P_{n-2}(x)-\eta_{n-2} P_{n-3}(x)\right) \\
& =\left(x-\epsilon_{n-1}\right) P_{n-1}(x)-a \epsilon_{n-1} P_{n-2}(x)+a\left(x-\epsilon_{n-2}\right) P_{n-2}(x)-a^{2} \epsilon_{n-2} P_{n-3}(x) \\
& =\left(x-\epsilon_{n-1}\right)\left(P_{n-1}(x)+a P_{n-2}(x)\right)-a \epsilon_{n-2}\left(P_{n-2}(x)+a P_{n-3}(x)\right) \\
& =\left(x-b_{n-1}\right) Q_{n-1}(x)-c_{n-1} Q_{n-2}(x) .
\end{aligned}
$$

Therefore, by Favard's theorem (see [3]), the sequence $\left\{Q_{n}(x)\right\}$ is the MOPS with respect to a regular linear functional or a positive definite functional.

In order to obtain that the sequence $\left\{Q_{n}(x)\right\}$ be a MOPS with respect to a positve Borel measure on $[-1,1]$, we can take, for example, $\epsilon_{n}=1 / 4$ and $a=1 / 16$. Then $b_{n}=1 / 4$ and $c_{n}=1 / 64$, and therefore the measure of orthogonality of the sequence $\left\{Q_{n}(x)\right\}$ belongs to the class $M(1 / 4,1 / 4)$, see [8]. Hence, the support of the measure is a subset of $[-1,1]$. 


\section{Linear combinations with constant coefficients of classical polynomials}

Jacobi polynomials. Now we consider the sequence of monic polynomials

$$
Q_{n+k}(x)=P_{n+k}^{(\alpha, \beta)}(x)+a_{1} P_{n+k-1}^{(\alpha, \beta)}(x)+\cdots+a_{k} P_{n}^{(\alpha, \beta)}(x), \quad n, k \geq 1,
$$

with $k$ a fixed integer, and $a_{i}(i=1, \ldots, k)$ real numbers such that $a_{k} \neq 0$. We continue denoting by $\left\{P_{n}^{(\alpha, \beta)}(x)\right\}$ the MOPS with respect to the Jacobi weight with parameters $\alpha$ and $\beta$.

Theorem 3.1. Let $\left\{Q_{n+k}(x)\right\}$ be the sequence defined in (3.1). If $\left\{Q_{n}(x)\right\}$ is the MOPS with respect to a linear regular functional or a positive finite measure on $[-1,1]$, then the sequence $\left\{P_{n}^{(\alpha, \beta)}(x)\right\}$ must be one of the four Chebyshev sequences, that is, $\alpha= \pm 1 / 2, \beta \pm 1 / 2$.

Proof. If $\left\{Q_{n}(x)\right\}_{n \geq 1}$ is a sequence of monic orthogonal polynomials, then there exist two sequences of real numbers $\left\{\epsilon_{n}\right\}$ and $\left\{\eta_{n}\right\}$, such that the following three-term recurrence relation holds, see [9],

$$
Q_{n+k}(x)=\left(x-\epsilon_{n+k-1}\right) Q_{n+k-1}(x)-\eta_{n+k-1} Q_{n+k-2}(x) .
$$

Taking into account relation (3.1), we can rewrite (3.2) as follows:

$$
\begin{aligned}
Q_{n+k}(x)= & \left(x-\epsilon_{n+k-1}\right)\left(P_{n+k-1}^{(\alpha, \beta)}(x)+\sum_{i=1}^{k} a_{i} P_{n+k-i-1}^{(\alpha, \beta)}(x)\right) \\
& -\eta_{n+k-1}\left(P_{n+k-2}^{(\alpha, \beta)}(x)+\sum_{i=1}^{k} a_{i} P_{n+k-i-2}^{(\alpha, \beta)}(x)\right) \\
= & \left(x-\epsilon_{n+k-1}\right) P_{n+k-1}^{(\alpha, \beta)}(x)+\left(\left(x-\epsilon_{n+k-1}\right) a_{1}-\eta_{n+k-1}\right) P_{n+k-2}^{(\alpha, \beta)}(x) \\
& +\sum_{i=2}^{k}\left(\left(x-\epsilon_{n+k-1}\right) a_{i}-\eta_{n+k-1} a_{i-1}\right) P_{n+k-i-1}^{(\alpha, \beta)}(x)-\eta_{n+k-1} a_{k} P_{n-2}^{(\alpha, \beta)}(x) .
\end{aligned}
$$

On the other hand, if we use the recurrence relation satisfied by the Jacobi polynomials, then (3.1) becomes

$$
\begin{aligned}
Q_{n+k}(x)= & \left(x-\beta_{n+k-1}\right) P_{n+k-1}^{(\alpha, \beta)}(x)-\gamma_{n+k-1} P_{n+k-2}^{(\alpha, \beta)}(x) \\
& +\sum_{i=1}^{k} a_{i}\left(\left(x-\beta_{n+k-i-1}\right) P_{n+k-i-1}^{(\alpha, \beta)}(x)-\gamma_{n+k-i-1} P_{n+k-i-2}^{(\alpha, \beta)}(x)\right) \\
= & \left(x-\beta_{n+k-1}\right) P_{n+k-1}^{(\alpha, \beta)}(x)+\left(a_{1}\left(x-\beta_{n+k-2}\right)-\gamma_{n+k-1}\right) P_{n+k-2}^{(\alpha, \beta)}(x) \\
& +\sum_{i=2}^{k}\left(\left(x-\beta_{n+k-i-1}\right) a_{i}-\gamma_{n+k-i} a_{i-1}\right) P_{n+k-i-1}^{(\alpha, \beta)}(x)-a_{k} \gamma_{n-1} P_{n-2}^{(\alpha, \beta)}(x) .
\end{aligned}
$$


By equating the second members of expressions (3.3) and (3.4), we get the following relations:

$$
\begin{aligned}
& \epsilon_{n+k-1}=\beta_{n+k-1}, \\
& a_{1} \epsilon_{n+k-1}+\eta_{n+k-1}=\gamma_{n+k-1}+a_{1} \beta_{n+k-2}, \\
& a_{2} \epsilon_{n+k-1}+a_{1} \eta_{n+k-1}= a_{1} \gamma_{n+k-2}+a_{2} \beta_{n+k-3}, \\
& \vdots \\
& a_{k} \epsilon_{n+k-1}+a_{k-1} \eta_{n+k-1}=a_{k-1} \gamma_{n}+a_{k} \beta_{n-1}, \\
& a_{k} \eta_{n+k-1}=a_{k} \gamma_{n-1} .
\end{aligned}
$$

If we substitute $\epsilon_{n+k-1}$ by $\beta_{n+k-1}$ and $\eta_{n+k-1}$ by $\gamma_{n-1}$, we can rewrite the other above equations using only the coefficients of the recurrence relation of the sequence $\left\{P_{n}^{(\alpha, \beta)}(x)\right\}$,

$$
\begin{gathered}
a_{1}\left(\beta_{n+k-1}-\beta_{n+k-2}\right)=\gamma_{n+k-1}-\gamma_{n-1}, \\
a_{2}\left(\beta_{n+k-1}-\beta_{n+k-3}\right)=a_{1}\left(\gamma_{n+k-2}-\gamma_{n-1}\right), \\
\vdots \\
a_{k}\left(\beta_{n+k-1}-\beta_{n-1}\right)=a_{k-1}\left(\gamma_{n}-\gamma_{n-1}\right), \quad \text { for } n \geq 2 .
\end{gathered}
$$

Now we can consider the following two cases.

(1) If $\beta_{n+k-1}=\beta_{n-1}$, then it must be $\alpha^{2}=\beta^{2}$, otherwise,

$$
(2 n+\alpha+\beta-2)(2 n+\alpha+\beta)=(2 n+2 k-2+\alpha+\beta)(2 n+2 k+\alpha+\beta),
$$

which is impossible unless $k=0$.

So we assume that $\alpha^{2}=\beta^{2}$.

We consider $i=\max \left\{j \in\{1, \ldots, k-1\}: a_{j} \neq 0\right\}$, or $i=0$ if $a_{j}=0$ for every $j=1, \ldots$, $k-1$. Then $\gamma_{n+k-(i+1)}-\gamma_{n-1}=0$, and we distinguish between two possibilities.

(i) If $\beta=\alpha$, the expression $\gamma_{n+k-(i+1)}=\gamma_{n-1}$ can be written as follows:

$$
\begin{aligned}
& \frac{4(n+k-i-1)(n+k-i-1+\alpha)^{2}(n+k-i-1+2 \alpha)}{(2 n+2 k-2 i-2+2 \alpha)^{2}(2 n+2 k-2 i-2+2 \alpha+1)(2 n+2 k-2 i-2+2 \alpha-1)} \\
& =\frac{4(n-1)(n-1+\alpha)^{2}(n-1+2 \alpha)}{(2 n-2+2 \alpha)^{2}(2 n+2 \alpha-1)(2 n+2 \alpha-3)},
\end{aligned}
$$

from which we obtain, after some computations,

$$
\begin{aligned}
& (n+k-i-1)(n+k-i-1+2 \alpha)(2 n+2 \alpha-1)(2 n+2 \alpha-3) \\
& \quad=(n-1)(n-1+2 \alpha)(2 n+2 k-2 i+2 \alpha-1)(2 n+2 k-2 i+2 \alpha-3) .
\end{aligned}
$$

It is easy to see that the coefficients of the powers $n^{4}, n^{3}$, and $n^{2}$ are equal in both members of the above formula. By equating the coefficients of $n$ and the 
independent terms, we get

$$
\begin{gathered}
\left(4 \alpha^{2}-1\right)(k-i)=0 \\
\left(4 \alpha^{2}-1\right)(k-i)(2 \alpha+k-i-2)=0 .
\end{gathered}
$$

Since $k-i \neq 0$, the system is compatible only for $\alpha^{2}=1 / 4$. Therefore, we obtain that $\alpha=\beta=1 / 2$ or $\alpha=\beta=-1 / 2$.

(ii) If $\beta=-\alpha$, the equality $\gamma_{n+k-(i+1)}=\gamma_{n-1}$ is

$$
\begin{aligned}
& \frac{4(n+k-i-1)(n+k-i-1+\alpha)(n+k-i-1-\alpha)(n+k-i-1)}{(2 n+2 k-2 i-2)^{2}(2 n+2 k-2 i-1)(2 n+2 k-2 i-3)} \\
& =\frac{4(n-1)(n-1+\alpha)(n-1-\alpha)(n-1)}{(2 n-2)^{2}(2 n-1)(2 n-3)},
\end{aligned}
$$

from which we deduce

$$
\begin{aligned}
& (n+k-i-1+\alpha)(n+k-i-1-\alpha)(2 n-1)(2 n-3) \\
& \quad=(2 n+2 k-2 i-1)(2 n+2 k-2 i-3)(n-1+\alpha)(n-1-\alpha) .
\end{aligned}
$$

Developing in powers of $n$, we see that the coefficients of $n^{4}, n^{3}$, and $n^{2}$ are the same in both members of the equality, and by equating the coefficients of $n$ and the independent term, it yields

$$
\begin{gathered}
\left(4 \alpha^{2}-1\right)(k-i)=0, \\
\left(4 \alpha^{2}-1\right)(k-i-2)=0,
\end{gathered}
$$

from which it follows that $\alpha^{2}=1 / 4$. Therefore, we obtain that $\alpha=-\beta=1 / 2$ or $\alpha=-\beta=-1 / 2$.

(2) If $\beta_{n+k-1} \neq \beta_{n-1}$, then $a_{k-1} \neq 0$ and $\gamma_{n}-\gamma_{n-1} \neq 0$. Therefore, from (3.6), we get $\gamma_{n}-$ $\gamma_{n-1}=\left(a_{k} / a_{k-1}\right)\left(\beta_{n+k-1}-\beta_{n-1}\right)$, for $n \geq 2$. If we take into account the expression of the coefficients in the recurrence relation for the Jacobi polynomials and we denote $R=$ $a_{k} / a_{k-1}$, we have

$$
\begin{aligned}
& \frac{4 n(n+\alpha)(n+\beta)(n+\alpha+\beta)}{(2 n+\alpha+\beta)^{2}(2 n+\alpha+\beta+1)(2 n+\alpha+\beta-1)}-\frac{4(n-1)(n-1+\alpha)(n-1+\beta)(n-1+\alpha+\beta)}{(2 n+\alpha+\beta-2)^{2}(2 n+\alpha+\beta-1)(2 n+\alpha+\beta-3)} \\
& =R\left(\beta^{2}-\alpha^{2}\right)\left(\frac{1}{(2 n+2 k+\alpha+\beta-2)(2 n+2 k+\alpha+\beta)}-\frac{1}{(2 n+\alpha+\beta-2)(2 n+\alpha+\beta)}\right),
\end{aligned}
$$


or equivalently

$$
\begin{aligned}
(2 n+\alpha+\beta+2 k-2)(2 n+\alpha+\beta+2 k) \\
\quad \times\left((2 n+\alpha+\beta-2)^{2}(2 n+\alpha+\beta-3) n(n+\alpha)(n+\beta)(n+\alpha+\beta)\right. \\
\left.\quad \quad-(2 n+\alpha+\beta)^{2}(2 n+\alpha+\beta+1)(n-1)(n-1+\alpha)(n-1+\beta)(n-1+\alpha+\beta)\right) \\
=R\left(\beta^{2}-\alpha^{2}\right)((2 n+\alpha+\beta-2)(2 n+\alpha+\beta)-(2 n+\alpha+\beta+2 k-2)(2 n+\alpha+\beta+2 k)) \\
\quad \times(2 n+\alpha+\beta)(2 n+\alpha+\beta+1)(2 n+\alpha+\beta-1)(2 n+\alpha+\beta-2)(2 n+\alpha+\beta-3) .
\end{aligned}
$$

Finally, we compute the coefficients of $n^{6}$ and $n^{5}$ obtaining the following system:

$$
\begin{gathered}
2 \alpha^{2}+2 \beta^{2}-1=-4 k R\left(\beta^{2}-\alpha^{2}\right), \\
\left(2 \alpha^{2}+2 \beta^{2}-1\right)(3 \alpha+3 \beta+2 k-3)=-2 k R\left(\beta^{2}-\alpha^{2}\right)(6 \alpha+6 \beta+k-6) .
\end{gathered}
$$

If we substitute the first equation in the second one, we obtain $k=0$, which is impossible. Therefore, case (2) cannot be satisfied.

Laguerre and Hermite polynomials. We consider the sequence of monic polynomials

$$
R_{n+k}(x)=L_{n+k}^{(\alpha)}(x)+a_{1} L_{n+k-1}^{(\alpha)}(x)+\cdots+a_{k} L_{n}^{(\alpha)}(x), \quad n, k \geq 1,
$$

with $k$ a fixed integer, and $a_{i}(i=1, \ldots, k)$ real numbers such that $a_{k} \neq 0$. We continue denoting by $\left\{L_{n}^{(\alpha)}(x)\right\}$ the MOPS with respect to the Laguerre weight with parameter $\alpha$.

We also consider the sequence of monic polynomials

$$
S_{n+k}(x)=H_{n+k}(x)+a_{1} H_{n+k-1}(x)+\cdots+a_{k} H_{n}(x), \quad n, k \geq 1,
$$

with $k$ a fixed integer, and $a_{i}(i=1, \ldots, k)$ real numbers such that $a_{k} \neq 0$. We continue denoting by $\left\{H_{n}(x)\right\}$ the MOPS with respect to the Hermite weight.

Theorem 3.2. Let $\left\{R_{n+k}(x)\right\}$ be the sequence defined in (3.17), and let $\left\{S_{n+k}(x)\right\}$ be the sequence defined in (3.18). Then the sequence $\left\{R_{n}(x)\right\}$ is not the MOPS with respect to any linear regular functional or positive finite measure on $[0,+\infty)$, and $\left\{S_{n}(x)\right\}$ is not the MOPS with respect to any linear regular functional or positive finite measure on $(-\infty,+\infty)$.

Proof. The idea is to solve the system (3.6) that appears in the previous theorem with the recurrence coefficients of the Laguerre and Hermite polynomials respectively. In the Laguerre case, if $\beta_{n+k-1}-\beta_{n-1}=0$, then we get $k=0$, which is impossible, and if $\beta_{n+k-1}-$ $\beta_{n-1} \neq 0$, we also get to a contradiction.

In the Hermite case, since $\beta_{n}=0$, we obtain $\gamma_{n+k-1}=\gamma_{n-1}$, which implies that $k=0$.

As a consequence we obtain the following corollary.

Corollary 3.3. Consider the sequence of monic polynomials

$$
Q_{n+k}(x)=P_{n+k}(x)+a_{1} P_{n+k-1}(x)+\cdots+a_{k} P_{n}(x), \quad n, k \geq 1,
$$


with $k$ a fixed integer, and $a_{i}(i=1, \ldots, k)$ real numbers such that $a_{k} \neq 0$, and $\left\{P_{n}(x)\right\}$ a classical MOPS (Jacobi, Laguerre, or Hermite). If $\left\{Q_{n}(x)\right\}$ is the MOPS with respect to a linear regular functional, then $\left\{P_{n}(x)\right\}$ must be one of the four Chebyshev orthogonal polynomial sequences.

\section{References}

[1] W. A. Al-Salam and A. Verma, Some sets of orthogonal polynomials, Rev. Técn. Fac. Ingr. Univ. Zulia 9 (1986), no. 2, 83-88.

[2] A. Branquinho and F. Marcellán, Generating new classes of orthogonal polynomials, Int. J. Math. Math. Sci. 19 (1996), no. 4, 643-656.

[3] T. S. Chihara, An Introduction to Orthogonal Polynomials, Mathematics and Its Applications, vol. 13, Gordon and Breach, New York, 1978.

[4] J. M. Garcia-Amor, Ortogonalidad Bernstein-Chebyshev en la recta real, Ph.D. thesis, Universidad de Vigo, Spain, 2003, (Spanish).

[5] Z. S. Grinshpun, Differential equations for the Bernstein-Szegö orthogonal polynomials, Differ. Equ. 26 (1990), no. 5, 545-550.

[6] Special linear combinations of orthogonal polynomials, J. Math. Anal. Appl. 299 (2004), no. $1,1-18$.

[7] K. H. Kwon, J. H. Lee, and F. Marcellán, Orthogonality of linear combinations of two orthogonal polynomial sequences, J. Comput. Appl. Math. 137 (2001), no. 1, 109-122.

[8] P. G. Nevai, Orthogonal polynomials, Mem. Amer. Math. Soc. 18 (1979), no. 213.

[9] G. Szegő, Orthogonal Polynomials, 4th ed., American Mathematical Society, Colloquium Publications, vol. 23, American Mathematical Society, Rhode Island, 1975.

[10] V. B. Uvarov, The connection between systems of polynomials orthogonal with respect to different distribution functions, U.S.S.R. Comput. Math. and Math. Phys. 9 (1969), no. 6, 25-36.

E. Berriochoa: Departamento de Matemática Aplicada I, Facultad de Ciencias de Ourense, Universidad de Vigo, 32004 Ourense, Spain

E-mail address: esnaola@uvigo.es

A. Cachafeiro: Departamento de Matemática Aplicada I, ETS Ingenieros Industriales, Universidad de Vigo, 36280 Vigo, Spain

E-mail address: acachafe@uvigo.es

J. M. Garcia-Amor: Departamento de Matemática, IES Gonzalo Torrente Ballester, 36003 Pontevedra, Spain

E-mail address: garciaamor@edu.xunta.es 


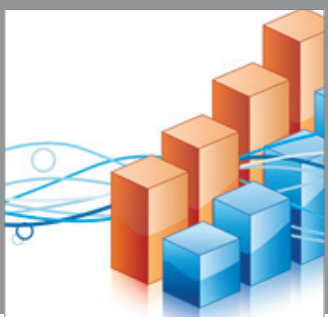

Advances in

Operations Research

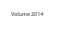

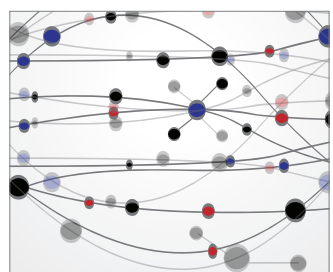

\section{The Scientific} World Journal
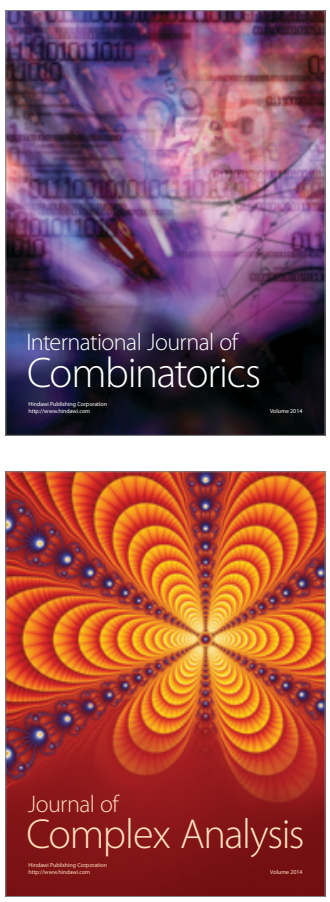

International Journal of

Mathematics and

Mathematical

Sciences
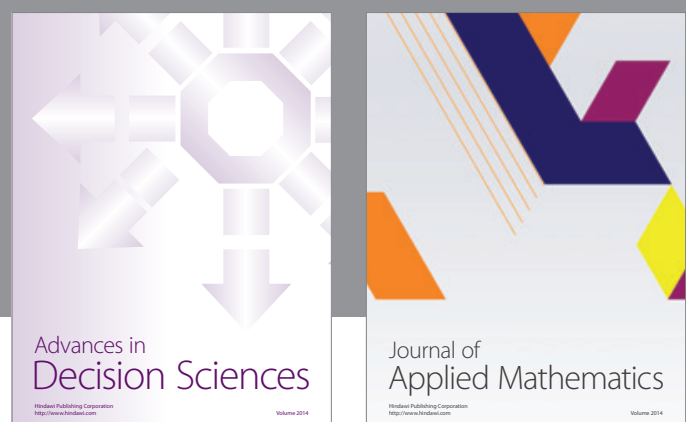

Journal of

Applied Mathematics
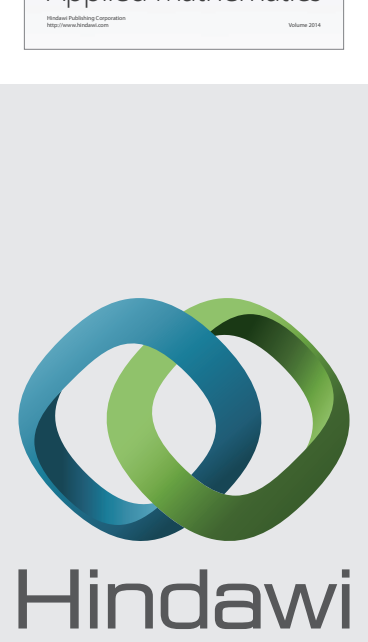

Submit your manuscripts at http://www.hindawi.com
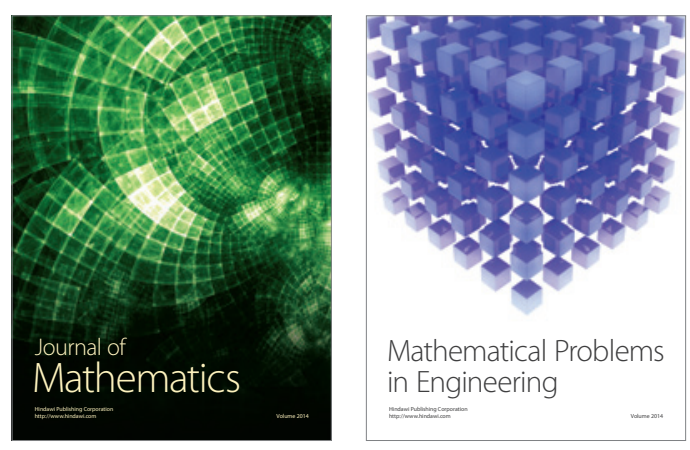

Mathematical Problems in Engineering
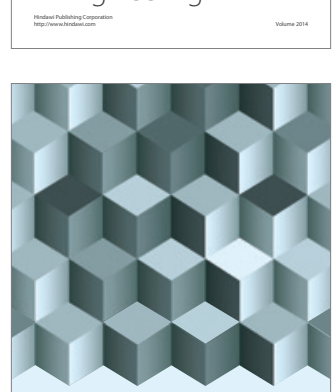

Journal of

Function Spaces
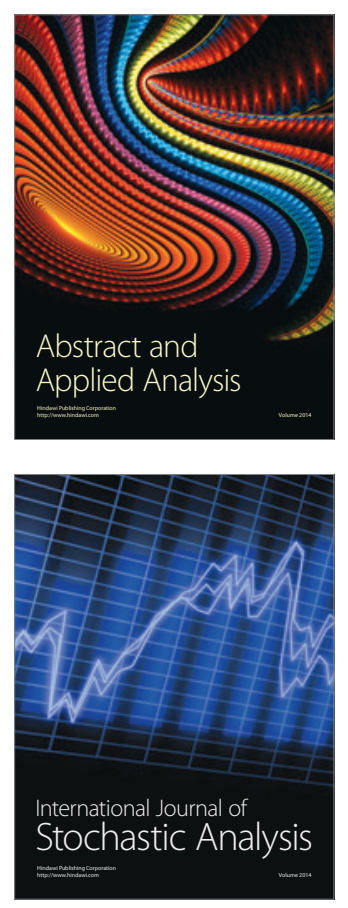

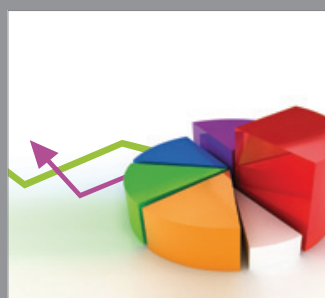

ournal of

Probability and Statistics

Promensencen
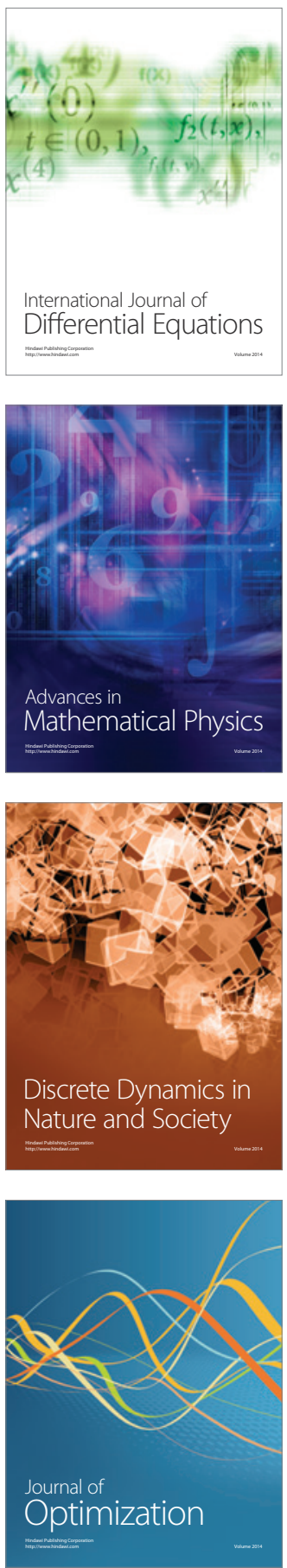